\title{
VLA radio observations of AR Scorpii ${ }^{\star}$
}

\author{
E. R. Stanway, T. R. Marsh, P. Chote, B. T. Gänsicke, D. Steeghs, and P. J. Wheatley
}

\author{
Department of Physics, University of Warwick, Gibbet Hill Road, Coventry CV4 7AL, UK \\ e-mail: e.r.stanway@warwick.ac.uk
}

Received 29 November 2017 / Accepted 22 January 2018

\begin{abstract}
Aims. AR Scorpii is unique amongst known white dwarf binaries in showing powerful pulsations extending to radio frequencies. Here we aim to investigate the multi-frequency radio emission of AR Sco in detail, in order to constrain its origin and emission mechanisms. Methods. We present interferometric radio frequency imaging of AR Sco at 1.5, 5 and $9 \mathrm{GHz}$, analysing the total flux and polarization behaviour of this source at high time resolution (10, 3 and $3 \mathrm{~s}$ ), across a full $3.6 \mathrm{~h}$ orbital period in each band.

Results. We find strong modulation of the radio flux on the orbital period and the orbital sideband of the white dwarf's spin period (also known as the "beat" period). This indicates that, like the optical flux, the radio flux arises predominantly from on or near the inner surface of the M-dwarf companion star. The beat-phase pulsations of AR Sco decrease in strength with decreasing frequency. They are strongest at $9 \mathrm{GHz}$ and at an orbital phase $\sim 0.5$. Unlike the optical emission from this source, radio emission from AR Sco shows weak linear polarization but very strong circular polarization, reaching $\sim 30 \%$ at an orbital phase $\sim 0.8$. We infer the probable existence of a non-relativistic cyclotron emission component, which dominates at low radio frequencies. Given the required magnetic fields, this also likely arises from on or near the M-dwarf.
\end{abstract}

Key words. white dwarfs - stars: variables: general - stars: individual: AR Scorpii - polarization

\section{Introduction}

AR Scorpii (hereafter AR Sco) is a white dwarf/M dwarf close binary with an orbital period of $3.6 \mathrm{~h}$ (Marsh et al. 2016). It shows strong pulsations in brightness associated with the $117.1 \mathrm{~s}$ spin period of its white dwarf, which, uniquely, are seen from the ultraviolet all the way to radio frequencies. The mismatch between spin and orbital periods suggest a relation with the intermediate polar class of white dwarf binaries in which an accreting magnetic white dwarf spins faster than the binary orbit due to accretion from a disc that is disrupted close to the white dwarf, where the magnetic field dominates (e.g. Wickramasinghe \& Meggitt 1982). However, the source shows little or no evidence for an accretion disc which might suggest a more natural match to the asynchronous polars, in which the difference between spin and orbital periods is likely attributable to an impulse from a nova event (Stockman et al. 1988). On the other hand, asynchronous polars typically present an orbit-spin period difference of less than 1\% (Warner 2002), and AR Sco is far from meeting that criterion. In fact, AR Sco is distinct from all known white dwarf/main sequence binary systems in a number of key respects, but primarily in the lack of any evidence for accretion and the great strength of its pulsations which are almost $100 \%$ modulated at ultraviolet wavelengths and which extend all the way to radio wavelengths.

Marsh et al. identified three dominant components in the frequency spectrum of AR Sco's flux variability: the orbital period of the binary around its barycentre $(3.56 \mathrm{~h})$, the spin period of the white dwarf (117.1 s) and the beat period between these (118.2 s).

\footnotetext{
* A table of the flux time series is only available at the CDS via anonymous ftp to cdsarc.u-strasbg.fr $(130.79 .128 .5)$ or via http://cdsarc.u-strasbg.fr/viz-bin/qcat?J/A+A/611/A66
}

Given the strength of the beat period, and the power-law spectral energy distribution extending from the optical to the radio, it was proposed that the source flux was dominated by synchrotron emission, arising from an interaction between the two components of the binary. Further, the lack of clear signatures of an accretion disk component in the spectrum, together with the energy requirements of the system, suggested that this emission was powered by energy liberated by the spin down of a magnetic, rapidly-rotating white dwarf.

AR Sco has since been the subject of intensive investigation and theoretical analysis. Littlefield et al. (2017), using optical data from Kepler and CRTS, refined the orbital parameters and demonstrated that while the orbital waveform is stable on timescales of $\sim 78$ days (the duration of the $K 2$ campaign), it alters slowly over timescales on the order of years, while also showing aperiodic variations superimposed on the regular periodic flux changes. Marcote et al. (2017) presented observations taken at $8.5 \mathrm{GHz}$ with the Australian Long Baseline Array (LBA) which confirmed that the radio emission originates from a compact point source, with no evidence for extended radio jets. Buckley et al. (2017) obtained polarimetric data in the optical, identifying very strong (40\%) spin-modulated linear polarization, and a few percent circular polarization, which also varies with time. These observations strengthen the case for interpretation of AR Sco as the first-known white dwarf pulsar, with $\sim 6 \%$ of the spin-down power reprocessed by magnetospheric interactions to generate a self-absorbed synchrotron spectrum that dominates from the radio to the optical.

The initial model proposed by Marsh et al. (2016) was of a rapidly rotating white dwarf, whose bipolar magnetic field "whips" past the tidally-locked red dwarf twice in every spin exciting emission from a hotspot on the face of the M-dwarf directed towards the white dwarf. The binary orbital motion 
then leads to a dominant modulation on the beat period. Geng et al. (2016) developed this model further, identifying AR Sco as a near-perpendicular rotator whose open field lines sweep through the M-dwarf's stellar wind, accelerating electrons in a bow shock region above the M-dwarf surface. They note that, in their near edge-on model, the hemisphere seen by the observer has magnetic field directions which cancel out, suggesting that little circular polarization should be seen. Katz (2017) has also developed a model for this system, suggesting that the white dwarf spin-down energy is instead dissipated through magnetic reconnection in the M-dwarf atmosphere, dubbing this process synchronization, and the system as a whole a synchronar. In this model AR Sco occupies a short-lived, transitional state lying between intermediate polars (which contain a rapidly rotating white dwarf in a binary with a tidally-locked red dwarf) and traditional polars (in which the white dwarf spin is magnetically locked to the orbital period: the field lines of the white dwarf interact with the field of the companion, and force the white dwarf into synchronous rotation). This model naturally accounts for shifts of the flux maximum away from mid-orbital phase due to precession of the magnetic poles, and for rapid flaring due to magnetic storms in the M-dwarf atmosphere. Given the complex and challenging task of modelling this source, additional data are useful for refining the interpretation.

While the radio observations presented by Marsh et al. (2016) confirmed the unusual nature of this source, they were limited to a single hour of data at low spatial resolution, and so could not constrain the orbital modulation in the radio. Similarly, those taken with the VLBI and presented by Marcote et al. (2017) confirmed AR Sco's identity as a point source and were able to recover an orbital variation lightcurve, but had neither the time resolution nor the frequency range to fully explore the properties of this source in the radio.

In this paper we present high time resolution radio data obtained with the Karl G. Jansky Very Large Array (VLA) at 1, 5 and $9 \mathrm{GHz}$ ( $L, C$ and $X$ bands respectively), and use this to explore the properties of AR Sco on different timescales. We present our data acquisition and reduction in Sect. 2. In Sect. 3 we consider the radio properties of AR Sco on timescales of the orbital period, while in Sect. 4 we identify and investigate pulsations on the system beat period. In Sect. 5 we investigate evidence for polarization in the source. In Sect. 6 we discuss and interpret the radio properties of AR Sco, before presenting our conclusions in Sect. 7.

\section{Data acquisition and reduction}

\subsection{Radio observations}

Radio observations of AR Scorpii were obtained at the Karl G. Jansky Very Large Array (VLA) between 2016 April 28 and 2016 May $2^{1}$. Data were taken at frequencies centred around $1.5 \mathrm{GHz}$ ( $L$ band, $6 \mathrm{~h}), 5 \mathrm{GHz}(C$ band, $4 \mathrm{~h})$ and $9 \mathrm{GHz}(X$ band, $4 \mathrm{~h}$ ), where the integration length was chosen to capture a full $3.6 \mathrm{~h}$ orbital period (see Table 1). Observations were taken with the phase centre at the source location. At 5 and $9 \mathrm{GHz}$, the visibilities were read out every $3 \mathrm{~s}$, while at $1.5 \mathrm{GHz}$ a readout interval of $1 \mathrm{~s}$ was selected. All observations were taken with the telescope in the $\mathrm{CnB}$-configuration. Full polarization information was recorded, and the standard broadband continuum correlator configuration adopted, with a frequency coverage of $1.5 \pm 0.5,5.0 \pm 1.0$ and $9.0 \pm 1.0 \mathrm{GHz}$. In the $1.5 \mathrm{GHz}$ band, two

1 Observations associated with programme VLA/16A-338, PI: Marsh.
Table 1. Observation summary.

\begin{tabular}{cccc}
\hline \hline Date & Observatory & Frequency & Int. time/s \\
\hline 2016 April 29 & W1m & $B G(5500 \AA)$ & 10010 \\
& & $Z(8800 \AA)$ & 9420 \\
2016 April 29 & VLA & $X(9.0 \mathrm{GHz})$ & 11700 \\
2016 May 1 & VLA & $L(1.5 \mathrm{GHz})$ & 16800 \\
2016 May 2 & VLA & $C(5.0 \mathrm{GHz})$ & 12000 \\
2016 May 4 & W1m & $B G(5500 \AA)$ & 4992 \\
& & $Z(8800 \AA)$ & 7525 \\
\hline
\end{tabular}

spectral windows (each of $64 \mathrm{MHz}$ bandwidth) were impossible to calibrate due to strong radio frequency interference (RFI) throughout the calibrator observations, and are omitted from the analysis. In all bands, RFI was flagged as required. Absolute flux and secondary phase calibration were performed using observations of standard calibrators 3C286 and J1626-2951 respectively.

Data were reduced (i.e. automatically flagged, bandpass, gain and flux calibrated) using the standard VLA data reduction pipeline, integrated in the Common Astronomy Software Applications (CASA, v4.5.2) package. This performs a reduction of continuum data in the Stokes $I$ parameter, calibrating total flux. Further flagging was performed where necessary (particularly in the $1.5 \mathrm{GHz}$ band) before subsets of the data were imaged, using the CLEAN task of CASA. A "Clark" cleaning algorithm was used, with a Briggs weighting parameter of 0.5. Images were constructed using a 1 arcsec sampling in the $1.5 \mathrm{GHz}$ band, and 0.75 arcsec sampling in the 5 and $9 \mathrm{GHz}$ bands.

AR Sco was clearly detected at all bands, even in very short integrations ( $10 \mathrm{~s}$ at $1.5 \mathrm{GHz}, 3 \mathrm{~s}$ at 5 and $9 \mathrm{GHz}$ ). All bands also show evidence for temporal variability on multiple timescales. Source fluxes were determined using the CASA IMFIT command on a region centred at the source location. In the 5 and $9 \mathrm{GHz}$ bands, the source was straightforwardly fit as a single object. In the $1.5 \mathrm{GHz}$ band, the flux from AR Sco is somewhat confused with a neighbouring source at a separation of 14.3 arcsec in RA and 1.2 arcsec in declination, which contributed substantially to the archival NVSS flux for AR Sco, as Fig. 1 demonstrates. The source separation is comparable to the synthesized beam of the VLA at this declination and frequency, and so may cause problems, particularly if the primary beam major axis is oriented east-west. Given that each subset of the data has a different $u v$-plane coverage, the severity of this issue varies and is considered on a case by case basis. In this band, the two objects were fit simultaneously as point sources. A good fit could be obtained in all cases.

The neighbouring object at RA \& Dec $16^{\mathrm{h}} 21^{\mathrm{m}} 46.26^{\mathrm{s}}-$ $22^{\circ} 53^{\prime} 11.86^{\prime \prime}$ (J2000) is consistent with a point source, with no significant temporal variability and a flux of $3.49 \pm 0.08 \mathrm{mJy}$ at $1.5 \mathrm{GHz}$.

We note that the strong variability of AR Sco can cause problems with image reconstruction from visibility data, and thus that IMFIT typically reports the system as being extended in long integrations, despite its point source nature (see Marcote et al. 2017). This is particularly true in the $9 \mathrm{GHz}$ band, and we use the integrated fit properties (i.e. the flux appropriate for an extended source) in this band, while treating AR Sco as a point source in the other bands. We have checked that this makes no significant difference to flux measurements at 1.5 and $5 \mathrm{GHz}$, but it corrects 


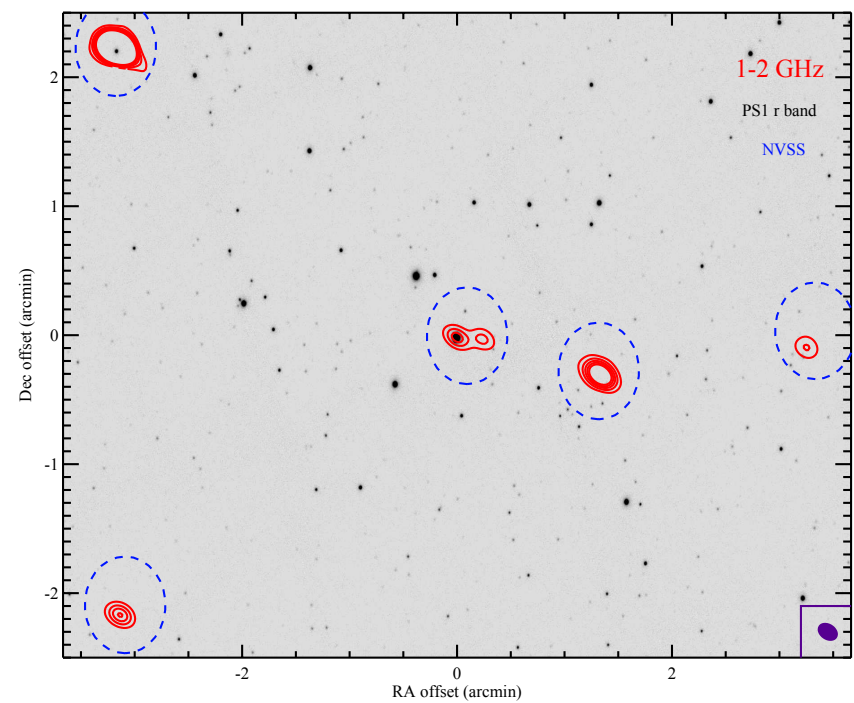

Fig. 1. The $1.5 \mathrm{GHz}$ radio environment of AR Sco. We show our radio contours, for an image constructed from a full orbital period, overlaid on the $r$-band image from the Pan-Starrs PS1 data release. Dashed ellipses show the sources identified in the $1.4 \mathrm{GHz}$ NRAO VLA Sky Survey (NVSS), at that survey's angular resolution. The presence of the neighbouring object to AR Sco (centred in the frame) and its contamination of the NVSS flux reported for this source, is clear. The synthesized beam of our $1.5 \mathrm{GHz}$ observations is shown in the lower right hand corner. Radio contours indicate multiples of $1.5 \mathrm{mJy} \mathrm{beam}^{-1}$.

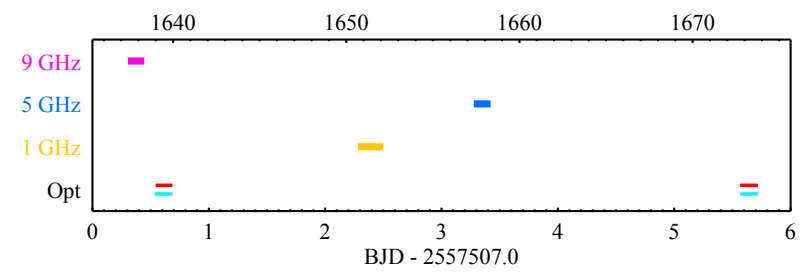

Fig. 2. Timeline of when observations were taken. Top axis indicates orbital intervals since the zero orbital phase defined in Marsh et al. (2016).

for the $30 \%$ of flux in the $9 \mathrm{GHz}$ band which would be lost if peak flux was considered instead.

\subsection{Optical observations}

To improve our constraints on source ephemerides and monitor for unusual behaviour in AR Sco, we obtained high time resolution, near-contemporaneous optical observations in two sessions which straddle the dates on which the VLA observed the target, as Fig. 2 illustrates. These observations were obtained using the dual-band high speed imager on the Warwick $1 \mathrm{~m}$ telescope (W1m) on La Palma. Each observation lasted $\sim 4 \mathrm{~h}$, and data were collected simultaneously in blue and red filters with integrations of $10 \mathrm{~s}$ on 2016 April 29 and of 2.5 and $5 \mathrm{~s}$ respectively on 2016 May 4. The blue filter has a wide optical bandpass, using BG40 glass, with peak efficiency at $\sim 5500 \AA$. The red filter is a $Z$-band, with peak throughput at $\sim 8800 \AA$. Due to the $3.3 \mathrm{~s}$ detector readout time between integrations, the $10 \mathrm{~s}$ integrations are more efficient in terms of on-sky observing time (see Table 1), while the shorter integrations capture more of the dynamic behaviour of this unusual source. Target flux calibration was performed relative to a nearby, non-variable point source to account for any possible seeing or sky brightness variations.

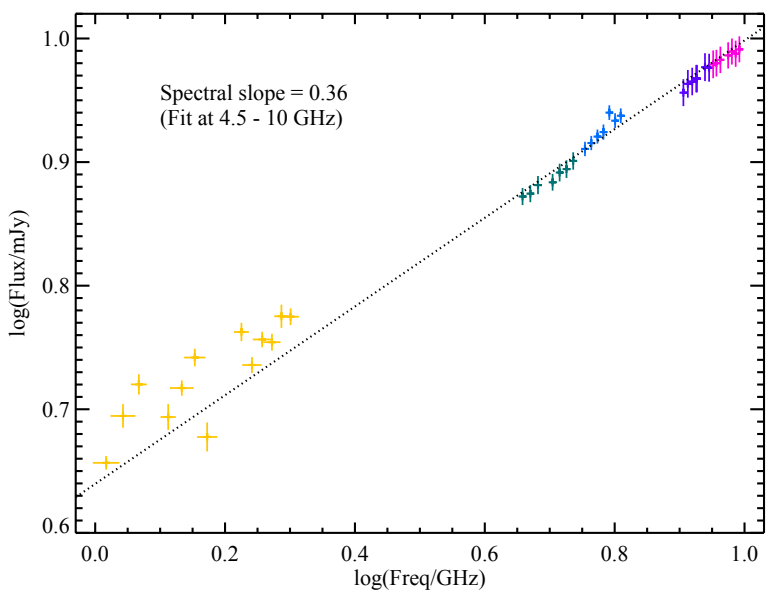

Fig. 3. Radio spectral energy distribution of AR Sco, averaged over a full orbital period. The dashed line indicates a fit in the form $F_{v} \propto v^{\alpha}$ to the spectral slope in the 5 and $9 \mathrm{GHz}$ bands, extrapolated to the $1.5 \mathrm{GHz}$ band. The best fit is found at $\alpha=0.358 \pm 0.015$.

\section{Orbital period properties}

\subsection{SED and spectral slope}

In order to determine the time-averaged spectral energy distribution of AR Sco, images were generated from a $3.6 \mathrm{~h}$ (i.e. full orbital period) interval of observations in each radio band. A secondary phase calibrator was visited every $10-15 \mathrm{~min}$, with approximately 2 min off-target on each occasion. As a result, the coverage is not fully continuous; the lacunae in the time series are randomly placed with respect to the orbit in each band.

A multifrequency synthesis covering this period was generated for each spectral window (i.e. each covering a bandwidth of $64 \mathrm{MHz}$ in the $1.5 \mathrm{GHz}$ band, $128 \mathrm{MHz}$ in the 5 and $9 \mathrm{GHz}$ bands), and the flux of AR Sco in each image determined. In Fig. 3 we show the orbit-averaged spectral energy distribution (SED) of AR Sco. Due to the lower signal to noise in the $1.5 \mathrm{GHz}$ band, and its different pulsation behaviour (see below), we do not use it to constrain the power law fitted to the higher frequency data. The data at 5 and $9 \mathrm{GHz}$ are consistent with a power law SED with $F_{\gamma} \propto v^{\alpha}$, where $\alpha=0.358 \pm 0.015$. While there is a hint that a slightly steeper power law may be appropriate within the $5 \mathrm{GHz}$ band, the overall fit is good, with an extrapolation of the high frequency power law contributing $>90 \%$ of the flux in the $1.5 \mathrm{GHz}$ band. The $4-10 \mathrm{GHz}$ power law fit is similar in spectral slope to the observed flux variation within both the 1.5 and $9 \mathrm{GHz}$ bands.

\subsection{Orbital variation}

In order to determine flux variability on the orbital period of the system, known from optical observations to be $3.6 \mathrm{~h}$, we image the field and measure the flux of AR Sco on intervals corresponding to individual on-source scans, between phase calibrator observations. These have a duration of $10 \mathrm{~min}$ in the 1.5 and $5 \mathrm{GHz}$ bands, and $15 \mathrm{~min}$ in the $9 \mathrm{GHz}$ band and so are sufficiently long to average over the $118 \mathrm{~s}$ spin/beat periods also known from the optical data. We image the data in $1 \mathrm{GHz}$ bandwidths centred at $1.5,4.5,5.5,8.5$ and $9.5 \mathrm{GHz}$ (i.e. splitting the $C$ and $X$ band observations into high and low frequency subbands).

In Fig. 4, we illustrate the frequency dependent total flux variability of AR Sco with orbital phase. These measurements 


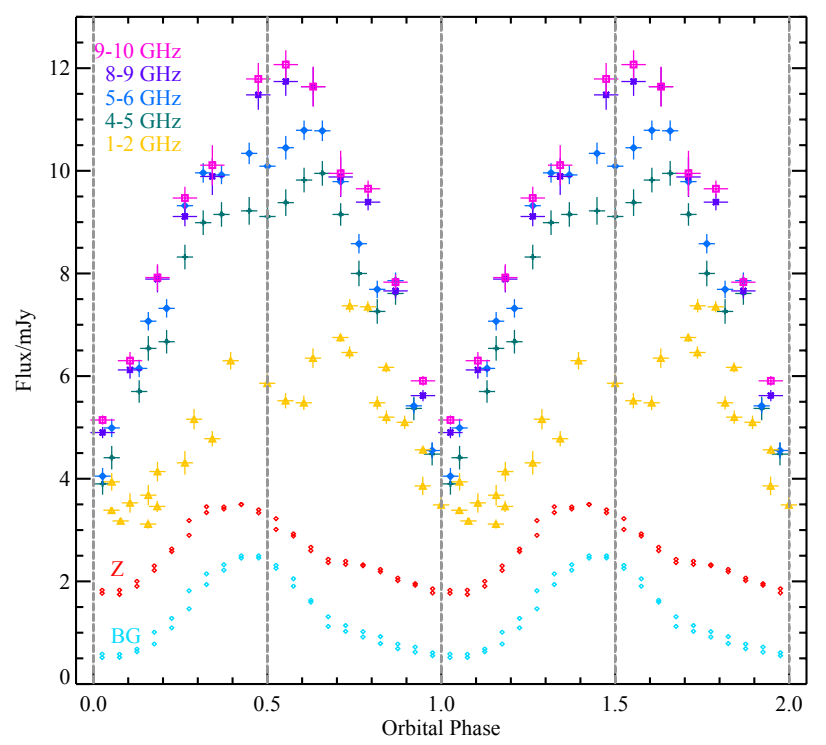

Fig. 4. Flux variation over the orbital period of AR Sco. Data points represents integration over a $10 \mathrm{~min}$ interval $(15 \mathrm{~min}$ at $9 \mathrm{GHz})$, sufficient to average over the 2 min beat period flux variation. Results are shown for $1 \mathrm{GHz}$ bands centred at $1.5,4.5,5.5,8.5$ and $9.5 \mathrm{GHz}$. The data are phase-folded in the interval between 0 and 1 , and repeated between 1 and 2 for clarity. For comparison we also show the W1m optical data as small points in the bottom two lines. The optical data in each observation have been phase folded and the mean in each phase bin calculated their fluxes are arbitrarily scaled to indicate orbital phase and shape in the optical. Where more than one point occurs at a given frequency and phase, it indicates measurements in different orbits.

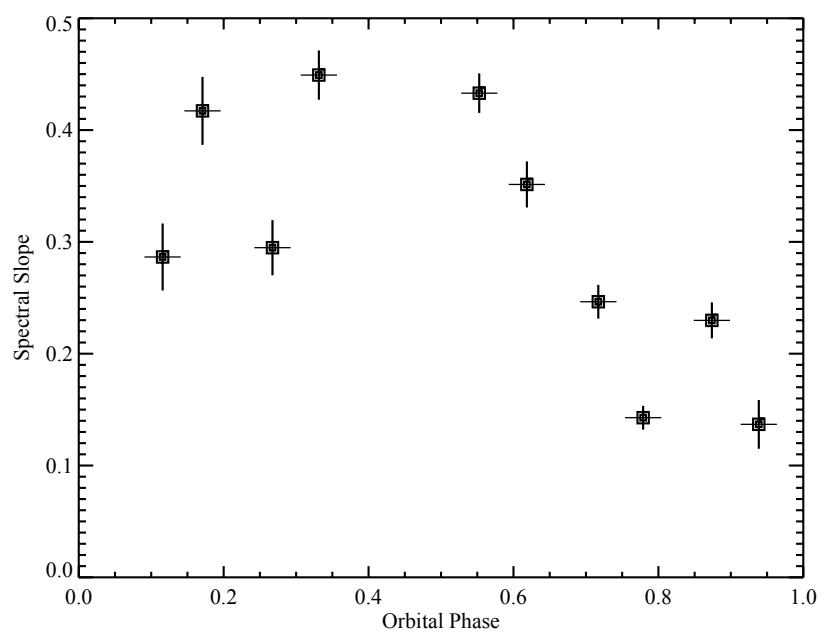

Fig. 5. Variation in 1-10 GHz spectral slope over the orbital period of AR Sco. Data points represent intervals of 0.05 in orbital phase for which data in all bands is available. In each case, a simple power law fit has been performed to the five bands defined in Fig. 4 .

are phase folded based on the median phase of each scan, using the zero-phase definition of Marsh et al. 2016 combined with the updated orbital period based on Kepler observations:

$T_{0}=57264.09615+0.148533 \times E$,

where $E$ is an orbit number and the time scale is TDB, corrected to the barycentre of the solar system, expressed as a Modified Julian Day number (BMJD = JD - 2400000.5 + barycentric correction). Zero phase is interpreted as the red dwarf lying closest to the observer along the line of sight. The optical data within each observation have been phase-folded and binned at 0.05 phase intervals with the mean at each phase, in each of the two observing epochs (2016-04-29 and 2016-05-02), plotted in the figure at an arbitrary flux level. The results are shown twice (i.e. repeated at phases between 1 and 2) to better illustrate the overall shape of the orbital flux variation.

As the figure makes clear, there is considerable variation in the shape of orbital flux variation with frequency. Both the radio data and the optical data reach minimum flux at or just after orbital phase zero. Both are also consistent with small variations in total flux from orbit to orbit, with phase-matched data taken in different orbits not always equal in amplitude. However their behaviour at peak (phase $=0.5$ ) and the overall shape of emission differs. The data at $8-10 \mathrm{GHz}$ shows a smooth variation in flux from peak to trough, with a sharply defined minimum and a much broader maximum in the light curve. The $4-6 \mathrm{GHz}$ data shows a smaller differential between the width of maximum and minimum, but a secondary dip in the flux, overlying the peak and occurring just before it in the lightcurve, appears and grows in strength as the frequency drops. The result is a double peaked light curve, with the first peak (at a phase of $\sim 0.35$ ) weaker than the second (at phase $\sim 0.65$ ). In the $1.5 \mathrm{GHz}$ band this behaviour is more pronounced, with the bottom of the light curve much broader and the peak heavily suppressed to leave a double-peaked curve.

As a result, the $1-10 \mathrm{GHz}$ spectral index of AR Sco, assuming a simple power law, also varies with orbital phase as Fig. 5 illustrates. Only those phases with data in all five subbands are fitted and shown. The spectral index decreases with increasing orbital phase, peaking at $\alpha \sim 0.5$ at an orbital phase of 0.5 (peak flux), before falling to $\sim 0.1$ at a phase of 1 (minimum flux).

The fractional flux variation on the orbital period, defined as $\left(f_{\max }-f_{\min }\right) /\left(f_{\max }+f_{\min }\right)$, is $0.41,0.44,0.45,0.41$ and 0.40 at $1.5,4.5,5.5,8.5$ and $9.5 \mathrm{GHz}$ respectively. Given the typical flux uncertainties and the probability that neither peak nor minimum flux are precisely sampled, this is consistent with a constant variation fraction with frequency.

\section{Beat period properties}

In order to explore the short time scale variation of AR Sco it is necessary to image the radio data, and measure the source flux, at very short intervals. Fortunately, the brightness of the source together with the very good $u v$-plane support for snapshot observations at the VLA allow this. We use a custom CASA python script to generate a CLEANed image and to run the IMFIT task on the visibility data for integration intervals of $3 \mathrm{~s}$ in the 5 and $9 \mathrm{GHz}$ bands and $10 \mathrm{~s}$ in the $1.5 \mathrm{GHz}$ band (where the source is relatively faint and the beam is large). In each case, the full bandwidth available $(2,2,1 \mathrm{GHz}$ respectively) is used. AR Sco is well detected in all these images and IMFIT reports that the source is a point source (as expected).

As before we fit AR Sco alone in the 5 and $9 \mathrm{GHz}$ bands, and the target and neighbouring source simultaneously in the $L$ band. In a handful of intervals, the fit failed to deconvolve the two sources, but in the majority the flux was successfully measured. The detailed lightcurve of AR Sco in each frequency band is shown in Fig. 6, and demonstrates the extraordinary variability of AR Sco on short timescales. This includes aperiodic flaring, particularly apparent in the $1.5 \mathrm{GHz}$ band, in addition to the known pulsations of the system. While the flares seen at $1.5 \mathrm{GHz}$ have not been reported in the optical, they show timescales of just a few minutes, and it is likely that a similar (20\% of total 
E. R. Stanway et al.: VLA radio observations of AR Scorpii
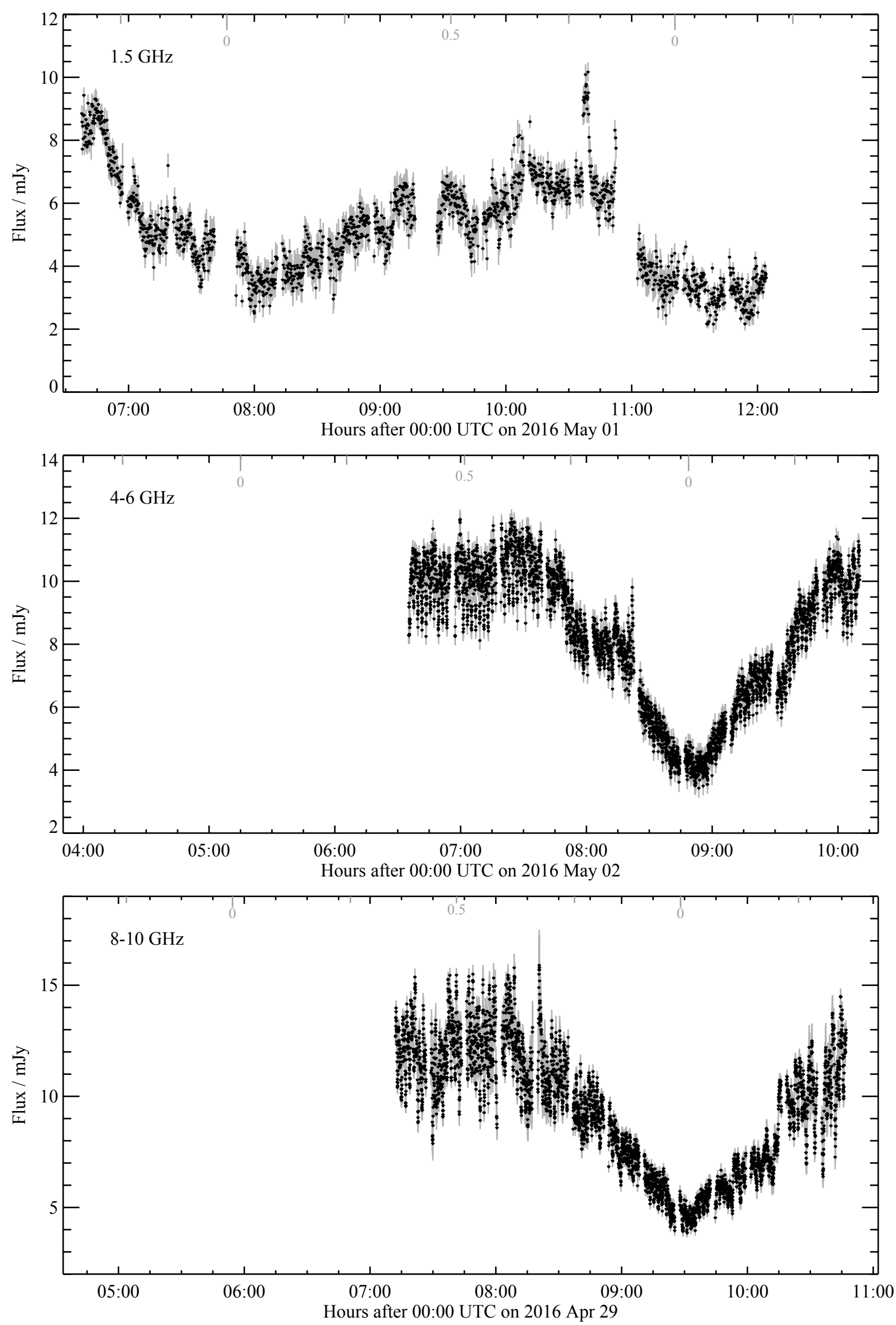

Fig. 6. Variation in the radio emission of AR Sco on short timescales. Each data point is a flux extracted from an image constructed from 10, 3 and $3 \mathrm{~s}$ integrations in the $1.5,5$ and $9 \mathrm{GHz}$ bands respectively. Orbital phase is marked above the lightcurves. 

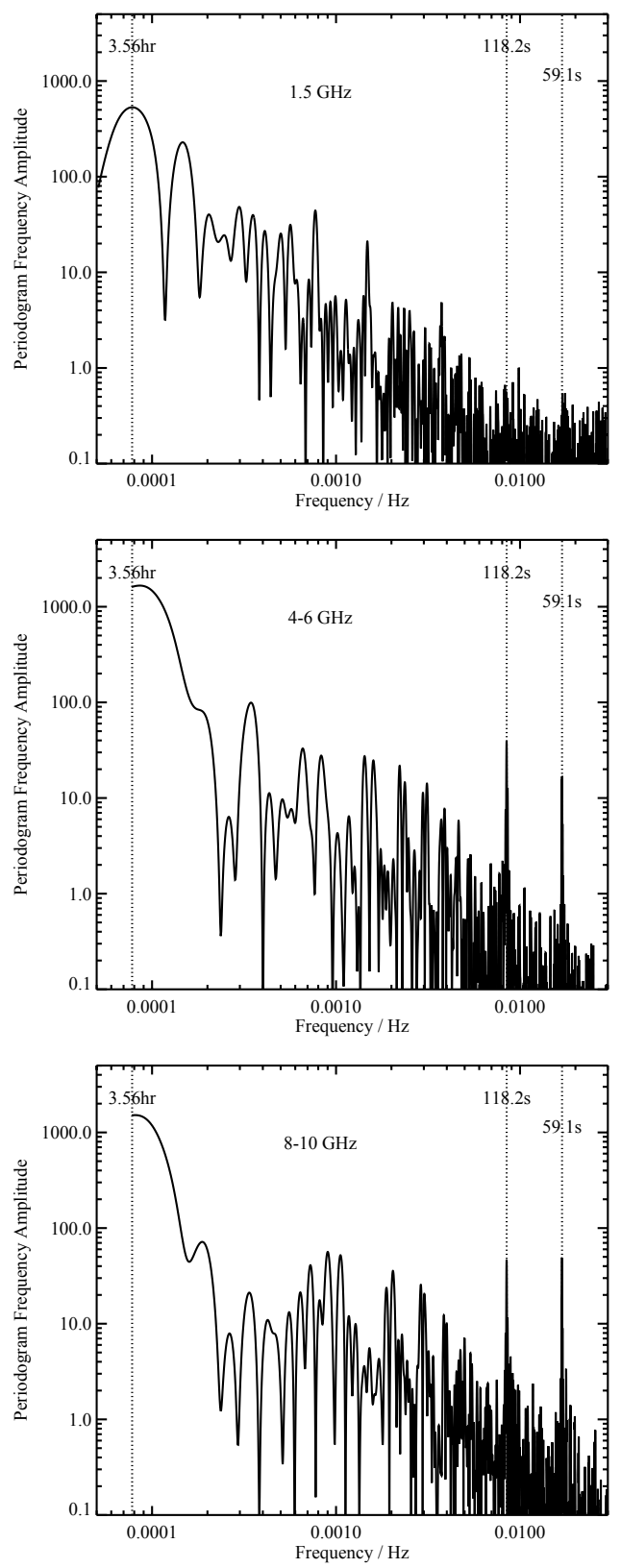

Fig. 7. Lomb-Scargle periodograms for the radio emission of AR Sco as a function of frequency. The orbital period $(3.56 \mathrm{~h})$, system beat period $(118.2 \mathrm{~s})$ and a half-beat period $(59.1 \mathrm{~s})$ are indicated by vertical lines.

flux) flare would be masked by the strong beat/spin modulation in the optical.

In Fig. 7 we calculate a Lomb-Scargle periodogram (Lomb 1976; Scargle 1982; Press \& Rybicki 1989) of each dataset, in order to determine the dominant frequency components in the variability. Unsurprisingly, there is a strong component at the orbital frequency in each band. There is also a signal at high frequencies. In Fig. 8, we examine this region, and twice its frequency, in more detail. At both 5 and $9 \mathrm{GHz}$ we identify significant power at a frequency consistent with the beat period $\left(P_{\mathrm{B}}=118.2 \mathrm{~s}\right)$ between the binary orbit and the white dwarf spin $\left(P_{\mathrm{S}}=117.0 \mathrm{~s}\right.$, Marsh et al. 2016). There is no clear evidence for emission on the spin period suggesting that the dominant power source for the radio emission lies in an interaction between the white dwarf and its red dwarf companion. The beat signal appears to decrease in strength with frequency. In the $1.5 \mathrm{GHz}$

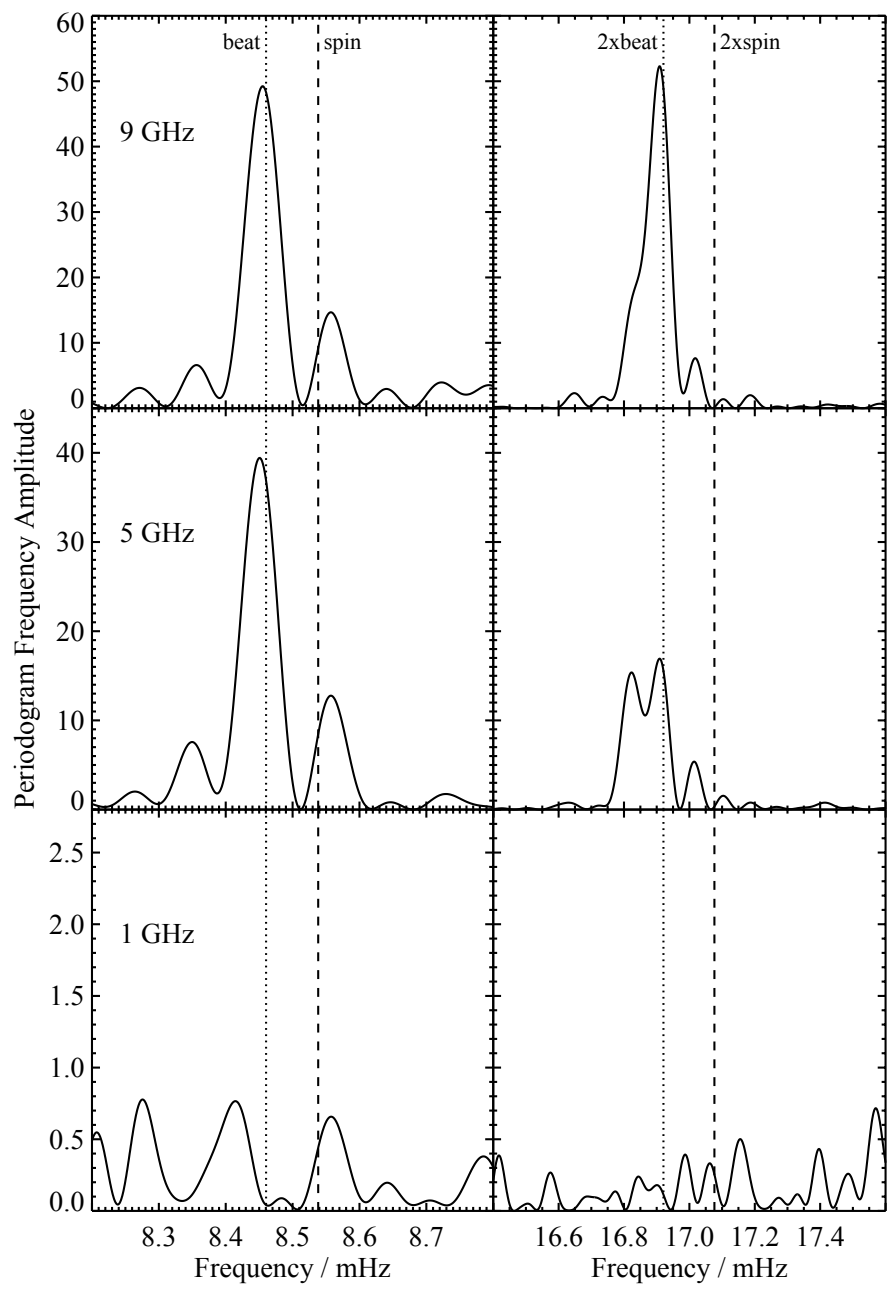

Fig. 8. Distinguishing beat from spin frequencies with the LombScargle periodograms for the radio emission of AR Sco as a function of frequency.

band, this beat signal disappears, and the only significant peak in the power spectrum corresponds to the orbital period, while the secondary peaks appear to be related to the length of individual scans with the VLA. To verify this, we have performed a test, subtracting a simple sinusoidal orbital modulation from the $1.5 \mathrm{GHz}$ data. The apparent periodogram features at about 10 and $20 \mathrm{~min}$ period are heavily suppressed in a periodogram of the residuals, confirming that they are associated with the window function of the data.

Phase-folding on the beat period, we are able to recover the beat-pulse lightcurve at each frequency. A reference zero beat phase is defined at $T_{0}$ (beat) $=57510.28387390$ (BMJD), which corresponds to a minimum at $5 \mathrm{GHz}$. This is shown in Fig. 9, where every datapoint has been normalised relative to the underlying orbital flux variation, determined by calculating a running mean across $450 \mathrm{~s}$ in each band. The plotted pulsation fraction is therefore defined by $f_{\mathrm{obs}, i} / f_{\text {mean }, \Delta i}$.

In the $5 \mathrm{GHz}$ and $9 \mathrm{GHz}$ bands, this pulsation fraction varies throughout the orbital period, reaching a maximum of about $\pm 20 \%$ with a $5 \%$ uncertainty. Taking the median value at each phase, the typical pulsation varies from +4 to $-8 \%$ around the mean flux in the beat cycle at $5 \mathrm{GHz}$ and from +8 to $-10 \%$ at $9 \mathrm{GHz}$. The beat oscillation is itself double peaked on the beat period, but asymmetric, with the two peaks of unequal strength. This behaviour was also seen in the near-infrared, optical and ultraviolet (see Marsh et al. 2016, Fig. 2). 

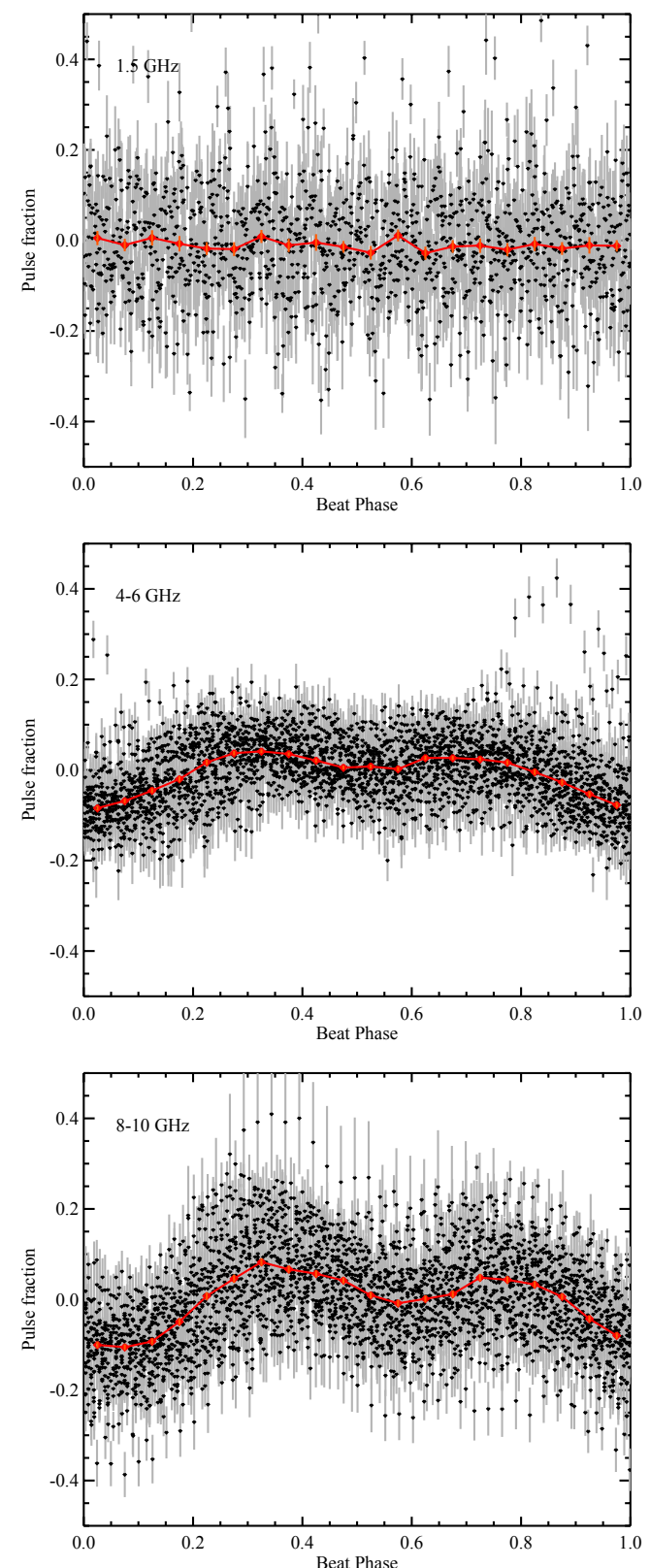

Fig. 9. Beat-folded lightcurves for the radio emission of AR Sco as a function of frequency. In each band, the data has been phase-folded by the $118.199 \mathrm{~s}$ beat period of the system, and each datum is normalised by a running mean over $450 \mathrm{~s}$. Red points show the median in beat period phase bins of 0.05 . Zero beat phase is defined at $T_{0}($ beat $)=57510.28387390(\mathrm{BMJD})$, which corresponds to a minimum at $5 \mathrm{GHz}$.

As Figs. 10 and 11 demonstrate, the same asymmetric double pulse within a beat period is identifiable in individual beat cycles at the peak of the orbital lightcurve, and is clearly detected at 5 and $9 \mathrm{GHz}$, with no apparent lag between them at a given beat phase. The ratio of the two pulses varies significantly from pulse to pulse, as exemplified by the example sequences of three consecutive beats at each frequency in Fig. 11. The two beats in each period are typically closer in strength at $5 \mathrm{GHz}$ than at $9 \mathrm{GHz}$, and not nearly so asymmetric as seen in the optical. There also appears to be a slight phase lag between the peaks at optical frequencies and those in the radio.

The pulsations fade with decreasing frequency, and cannot be clearly seen in the $1.5 \mathrm{GHz}$ data, even at peak orbital flux. At the
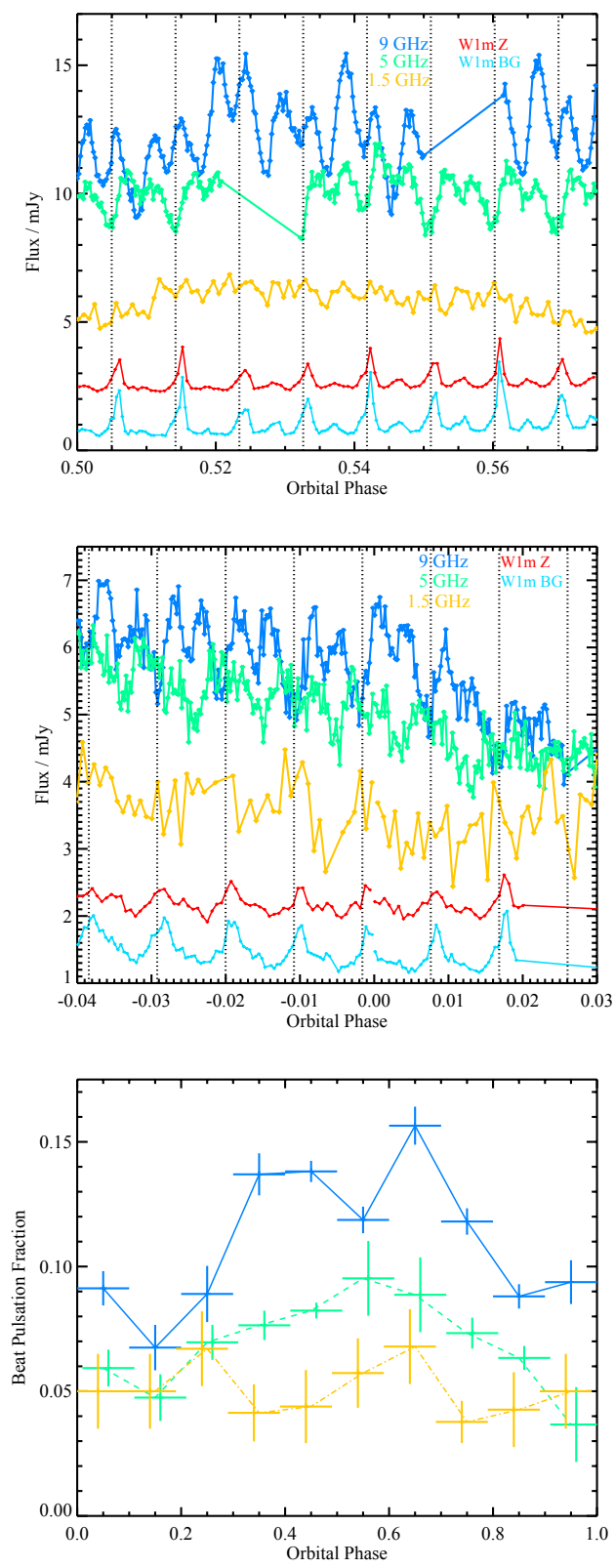

Fig. 10. Dependence of beat strength on orbital phase and frequency. We show that the beat pattern is captured in short integrations at the peak of the orbital lightcurve and at the orbital minimum. Vertical dotted lines mark intervals of $118.2 \mathrm{~s}$ (the beat period), referenced to the $5 \mathrm{GHz}$ data as in Fig. 9. Optical data from W1m are shown with an arbitrary flux scaling for comparison. In the bottom panel, we indicate the halfamplitude of the phase-folded and binned beat lightcurve in each orbital phase bin, at $1.5,5$ and $9 \mathrm{GHz}$.

minimum of the orbital lightcurve (orbital phase $=0$ ), it becomes harder to identify pulsations at all frequencies, primarily due to reduced signal to noise in the individual integrations. By subdividing the data first on orbital phase, and then on beat phase, and calculating the median in 0.05 beat phase bins (as in Fig. 9), it is possible to look at how the pulsation strength varies with orbital phase, and we show this in the bottom panel of Fig. 10. Here we use the half-difference between the maximum and minimum in the orbit- and beat-folded, median-averaged lightcurve as a proxy for pulsation strength. The strongest pulsations, varying by $15 \%$ relative to the median flux in a 0.1 orbital phase (21.4 min) bin, are seen in the $9 \mathrm{GHz}$ data at an orbital phase around $0.5-0.6$. The $5 \mathrm{GHz}$ data shows a similar strengthening of 


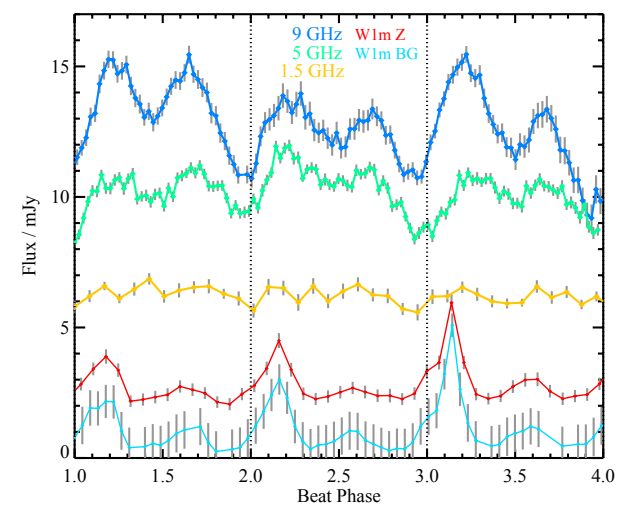

Fig. 11. The dependence of beat strength on beat phase and frequency. For each band, we show three consecutive beat cycles, extracted near the peak of the orbital light-curve and phased on the beat ephemeris. Only the $\mathrm{W} 1 \mathrm{~m} B G$ and $Z$ bands are simultaneous, and these have an arbitrary scaling in flux, for comparison with the radio data. The significant beatto-beat variations in the emission are apparent, as is a slight offset in beat phase between the optical and radio bands. Colour coding is as in Fig. 10.

the pulse fraction at an orbital phase of $\sim 0.5$. The $1.5 \mathrm{GHz}$ data do not show any evidence for periodic variation in the folded lightcurves, and the typical $10 \%$ difference between maximum and minimum in these lightcurves reflects the signal to noise of the data.

\section{Polarization}

In addition to total flux measurements (the Stokes I parameter), the VLA data contains full polarization information. Observations were taken at each frequency of the low-polarization leakage calibrator J1407+2827 as part of the relevant scheduling block. These were used to determine the instrumental polarization, while the known polarization properties of our primary flux calibrator 3C286 were used to determine the cross-hand delays and the $R-L$ polarization angle. Standard CASA tasks GAINCAL and POLCAL were used to calculate these, and they were applied together with the basic gain, delay and flux amplitude calibration tables generated by the VLA pipeline.

\subsection{Polarization with orbital phase}

\subsection{1. $9 \mathrm{GHz}$ band}

We first explore polarization data at $9 \mathrm{GHz}$ ( $X$ band), where the total flux and beat signal are strongest. In Fig. 12 we illustrate the polarization with orbital phase. Data were initially imaged by $(15 \mathrm{~min}) \mathrm{scan}$ in all four Stokes parameters. Once the variation was identified in the Stokes $V$ parameter, each scan was divided into two 6 min periods (where an even number of minutes was chosen to ensure consistent averaging over the beat period). Note, due to the necessity of phase calibration and the non-integer number of beat periods per scan, there is an unobserved interval between each pair of integrations. As before, the CASA IMFIT function was used to determine the flux of AR Sco in each image, and where the fit failed due to the lack of any visible source, a flux of zero is assigned in that Stokes parameter. Typical uncertainties are of order $0.2 \%$ in the polarization, dominated by the RMS uncertainty in the polarized images, rather than the total Stokes I flux.

The resultant polarization light curve shows a strong dependence on orbital phase. The linear polarization is consistently
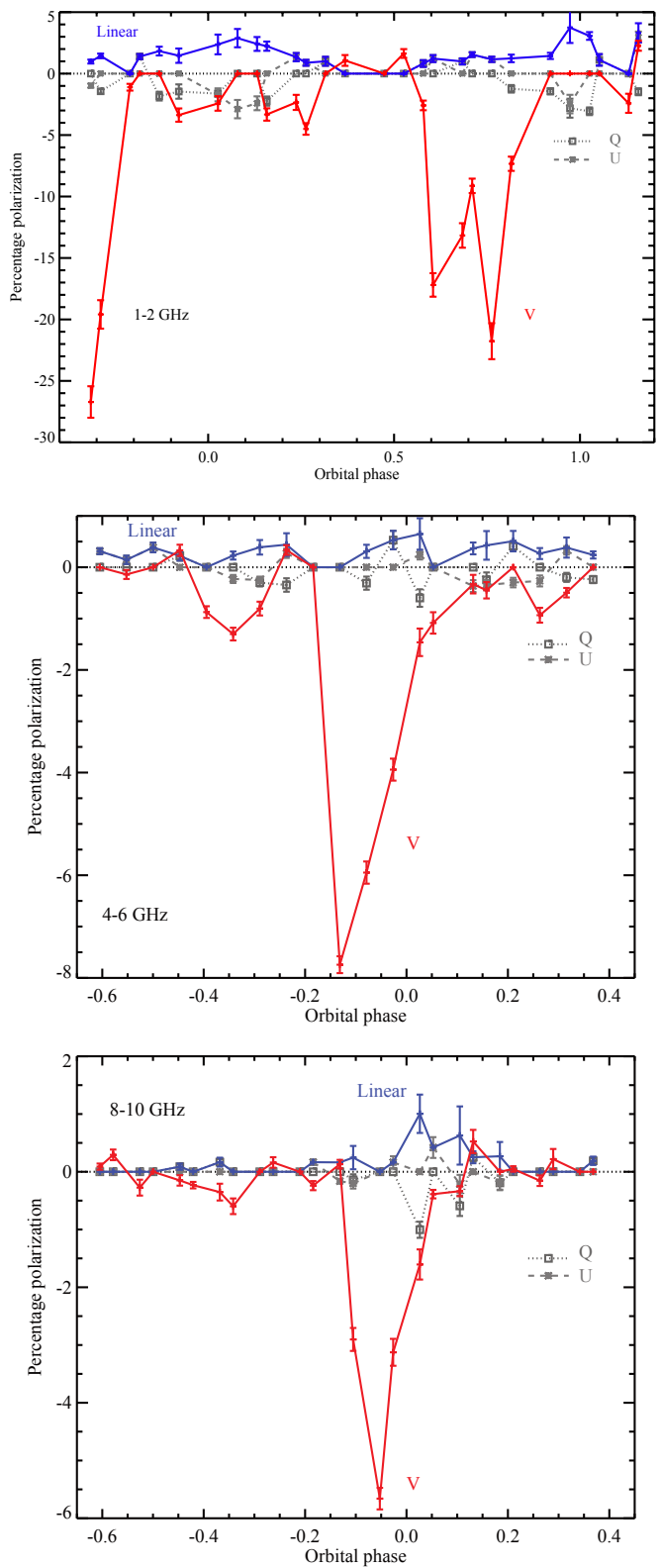

Fig. 12. The dependence of polarization on orbital phase at $1.5 \mathrm{GHz}$ (upper panel), $5 \mathrm{GHz}$ (middle panel) and $9 \mathrm{GHz}$ (lower panel). Data were imaged in all four Stokes parameters in 10, 10 and 6 min time bins respectively (i.e. averaging over beat period behaviour). We show the circular polarization percentage (Stokes $V / I$ ) and the total linear polarization percentage $\left(\sqrt{\left(Q^{2}+U^{2}\right) / I^{2}}\right)$, as well as the Stokes $Q$ and $U$ terms. An identically zero polarization indicates that IMFIT was unable to identify a source at the location of AR Sco in that Stokes parameter.

low, with either no detectable source in the Stokes parameter imaging or a point source detection at $<2 \sigma$ over the bulk of the orbital period. However at orbital phases between $-0.05(0.95)$ and 0.2 , the linear polarization is consistently above zero (albeit at low significance). The dominant component switches between $Q$ and $U$, suggesting a rapid rotation of the linear polarization (on a timescale of minutes). At its maximum, the source has a total linear polarization (in a 6 min interval) of $1.0 \pm 0.3 \%$.

By contrast both the strength and variation of circular polarization (the Stokes $V$ parameter) in AR Sco are more extreme. For the bulk of the orbital period, the source is consistent with exhibiting negative (anticlockwise) circular polarization. This polarization becomes highly significant at an orbital phase of 

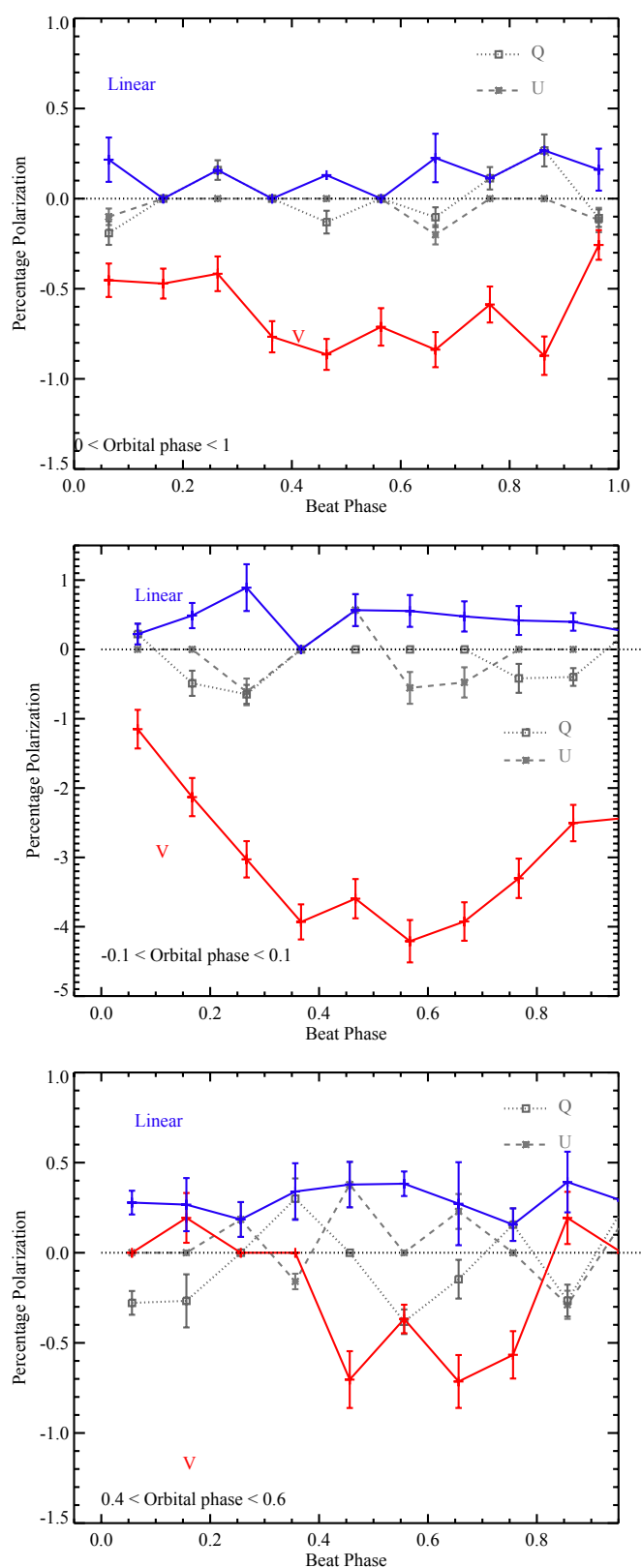

Fig. 13. The dependence of polarization on orbital phase and beat phase at $9 \mathrm{GHz}$. Visibility data were folded on the beat period, and each bin of 0.1 in beat phase separately imaged in $I Q U V$. Beat phase zero is defined as in Fig. 9. This procedure was repeated for two subsets at orbital phases $\approx 0$ and 1 . An identically zero polarization indicates that IMFIT was unable to identify a source at the location of AR Sco in that Stokes parameter.

$\approx 0.95$ with $V=-5.7 \pm 0.2 \%$. It exhibits a secondary (lower significance) negative peak with $V=-0.23 \pm 0.06 \%$ at an orbital phase of $\approx 0.65$.

\subsection{2. $5 \mathrm{GHz}$ band}

This behaviour is mirrored in the polarization at $5 \mathrm{GHz}$ ( $C$ band), where we integrate over individual $10 \mathrm{~min}$ scans. As before, the source is consistent with a very low linear polarization, peaking short of $1 \%$ at an orbital phase close to zero. By contrast, the circular polarization shows a very strong orbital modulation, peaking at $V=-7.7 \pm 0.3 \%$, at an orbital phase of 0.9 , somewhat earlier than that seen at $9 \mathrm{GHz}$. The secondary peak, at an orbital
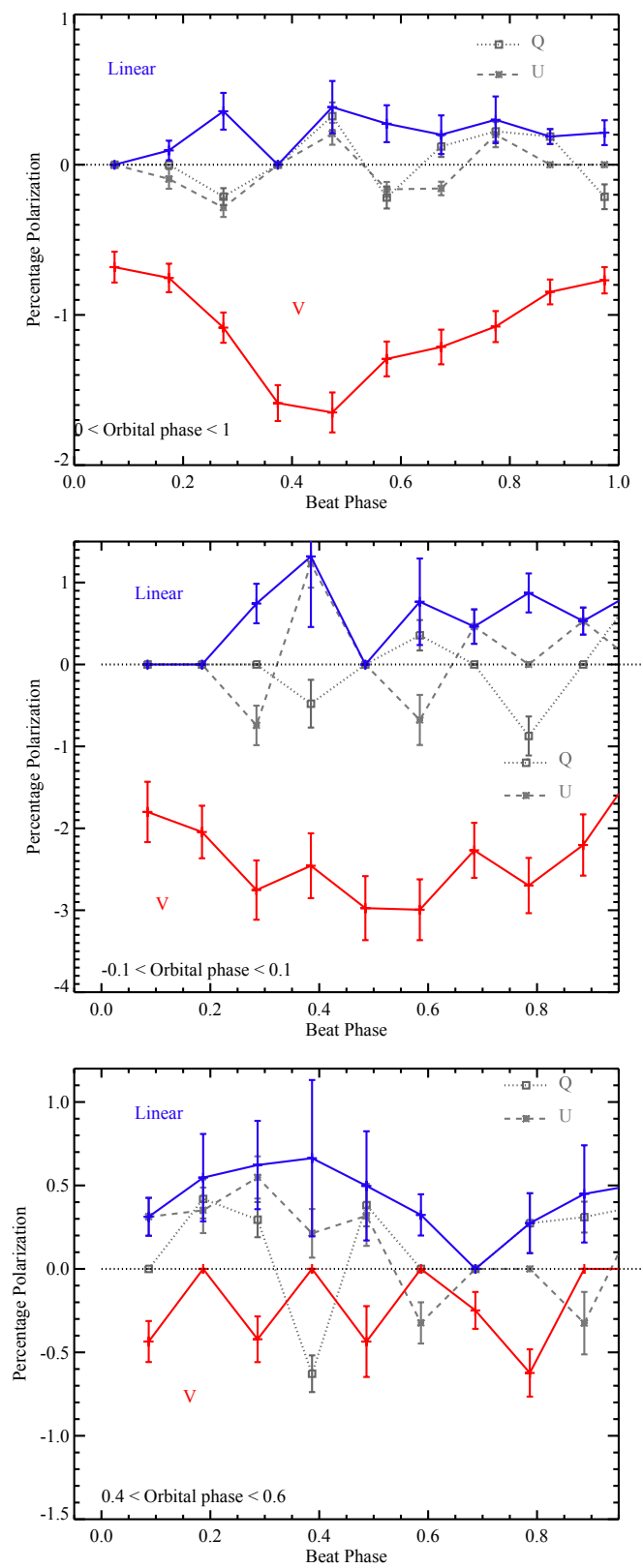

Fig. 14. The dependence of polarization on orbital phase and beat phase at $5 \mathrm{GHz}$. As in Fig. 13.

phase of 0.65 , is also reproduced and is well-detected, reaching $V=-1.3 \pm 0.2 \%$.

\subsection{3. $1.5 \mathrm{GHz}$ band}

The orbital behaviour of polarization at $1.5 \mathrm{GHz}$ ( $L$ band) is far more extreme than that seen at higher frequencies. Again we integrate over $10 \mathrm{~min}$ scans, and do not attempt to subdivide these due to the relatively low total flux signal-to-noise in this band. The lightcurve at $1.5 \mathrm{GHz}$ spans more than a full orbital period, giving more indication of the orbit-to-orbit variation. The linear polarization is in the range $1-3 \%$ for the majority of the orbit, reaching a minimum around an orbital phase of 0.45 and showing a maximum of $\approx 3 \%$, close to an orbital phase of 1 (slightly after this in the first orbit, slightly before in the second, but consistent given the random uncertainties on each data point). 

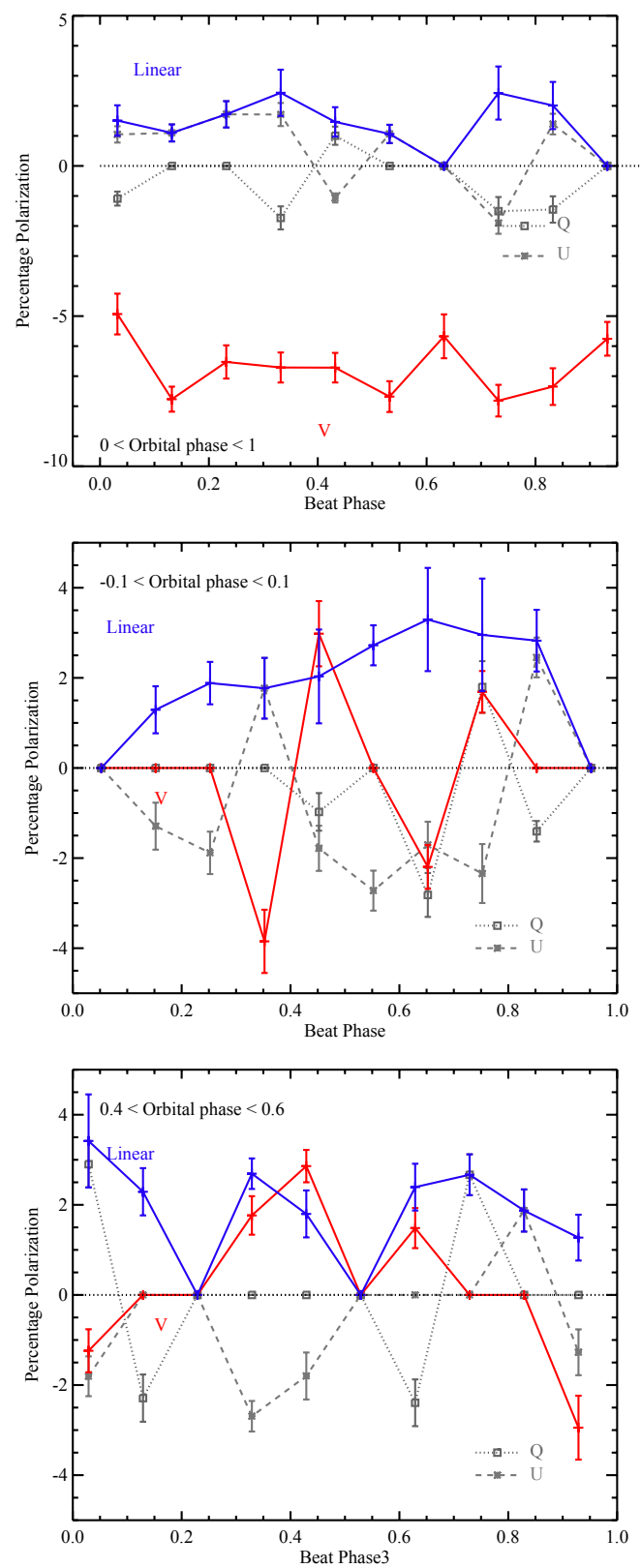

Fig. 15. The dependence of polarization on orbital phase and beat phase at $1.5 \mathrm{GHz}$. As in Fig. 13.

The circular polarization shows a similar excursion to large negative values to that seen at higher frequencies, but reaching $V=-27 \pm 1 \%$ in the first orbit and $V=-22 \pm 1 \%$ in the second orbit. This negative peak appeared earlier at 5 than $9 \mathrm{GHz}$. It appears earlier still at $1.5 \mathrm{GHz}$ and is broader, with a significant negative excursion in polarization between phases of 0.6 and 0.8 . This feature also shows internal structure, splitting into two overlapping negative peaks, rather than being a single narrow peak as seen at higher frequencies. The secondary peak seen at 5 and $9 \mathrm{GHz}$ is also apparent at $1.5 \mathrm{GHz}$, again shifted earlier in the orbit.

\subsection{Polarization with beat phase}

\subsection{1. $9 \mathrm{GHz}$ band}

In optical light, the polarization of AR Sco is known to vary strongly on the beat and spin periods (Buckley et al. 2017).
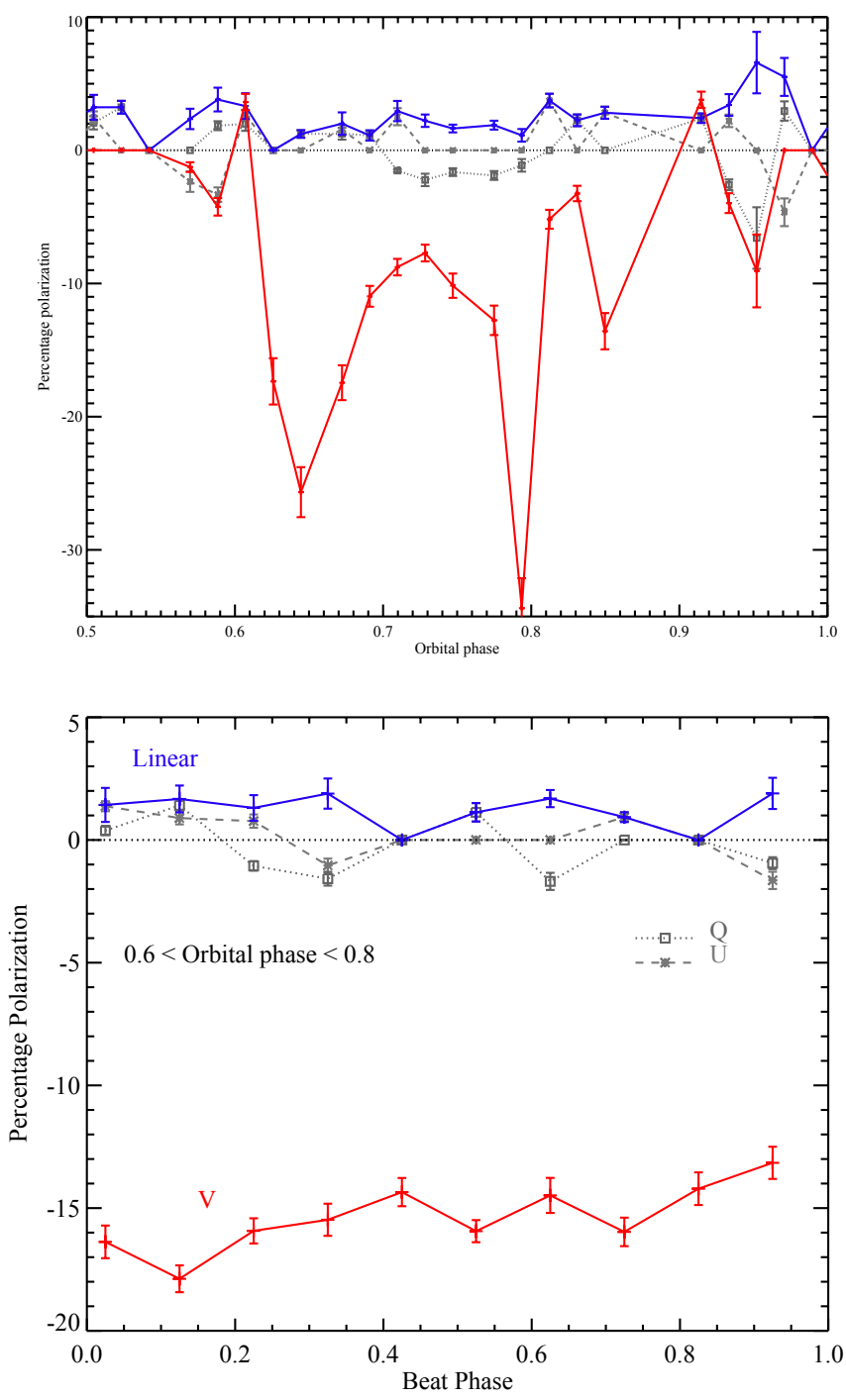

Fig. 16. Closer study of the negative circular polarization excursion of the $1.5 \mathrm{GHz}$ orbital lightcurve, now imaged in 4 min intervals, rather than $10 \mathrm{~min}$. In the lower panel we show the dependence of polarization on beat phase during this excursion.

In the radio, we lack the signal-to-noise and $u v$-plane coverage to resolve the polarization on these 2 min periods. Instead we investigate behaviour using phase folding as before. Individual visibility integrations were assigned a beat phase, based on the $118.2 \mathrm{~s}$ beat period and referenced to the same $(5 \mathrm{GHz}$ beat minimum) zero phase as in Sect. 4. The visibilities were then grouped by this beat phase, and an image created in each Stokes parameter, including only those visibilities contributing to a given beat phase bin. This was done for the entire observation (i.e. a full orbital period) and separately for subsets of the data covering $20 \%$ in orbital phase, centred near the maximum and minimum of the orbital flux variation. The resulting polarization light curves are shown in Fig. 13.

As is clear from the figures, the very low linear polarization at $9 \mathrm{GHz}$, in contrast to the $40 \%$ polarization seen in the optical, does not arise entirely from the effects of averaging over the beat cycle. Splitting the data by beat phase suggests that the linear polarization is nearly constant through the beat cycle, at a low level with no clear dependence on beat phase. There is also no clear dependence of the linear polarization beatperiod lightcurve on orbital phase. The mean linear polarization 


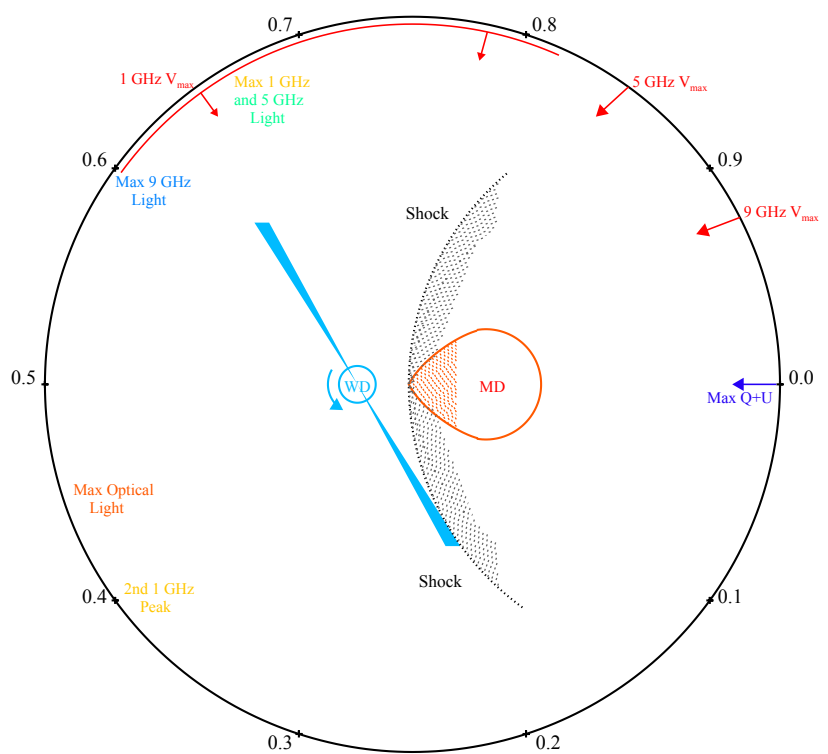

Fig. 17. Orbital phase diagram for the AR Sco system (not to scale). Key phases at which the system output peaks are indicated. We also show the beamed emission proposed for the white dwarf and potential shocks where it meets the white dwarf magnetosphere.

in the upper panel of Fig. 13 (i.e. dividing by beat phase for the whole orbital period) is $0.16 \pm 0.03 \%$. However it is possible that this is averaging out some of the signal. When the data for the two subsets in the lower panels of Fig. 13 are considered, the mean $9 \mathrm{GHz}$ linear polarization increases to $0.37 \pm 0.04 \%$. This suggests that there are short intervals with significantly higher polarization than the mean. If the polarization angle varies (i.e. signal is exchanged between the Stokes $Q$ and $U$ parameters), such short-term polarization signals would be washed out in a time-averaged flux. There is some hint of this happening - while the total linear polarization remains broadly constant through the beat period, both $Q$ and $U$ fluxes vary significantly in adjacent phase bins.

By contrast, the $9 \mathrm{GHz}$ circular polarization, shown in Fig. 12 to be strongly dependent on orbital phase, also appears to be sensitive to beat phase. The Stokes $V$ parameter shows negative flux (i.e. counterclockwise circular polarization in the signal), at all beat phases when averaging over the orbit, or when considering the minimum of the Stokes $I$ lightcurve. It is strongest at a beat phase of 0.5 (where zero marks the minimum total flux), peaking at $V=-4.2 \pm 0.3 \%$.

Finally, there is no clear evidence for polarization variation when folding on the spin (rather than beat) frequency. Reproducing Fig. 13 on the spin period yields near-constant linear polarization at the same level $(\sim 0.4 \%)$, while circular polarization shows a similar strength and pattern to that folded on the beat period (i.e. appearing strongest, with $V \sim-4 \%$, at the minimum of the folded light curve).

\subsection{2. $5 \mathrm{GHz}$ band}

As before, we repeat our analysis at $5 \mathrm{GHz}$, showing the results in Fig. 14. Beat period polarization modulation at this frequency mirrors that at $9 \mathrm{GHz}$. The linear polarization does not exceed $1 \%$ at any beat phase (when phase-folded) but is typically nonzero. The circular polarization is again modulated on the beatphase, peaking at a beat phase of $0.4-0.5$.

\subsection{3. $1.5 \mathrm{GHz}$ band}

The results of folding the $1.5 \mathrm{GHz}$ data on the beat period are shown in Fig. 15. These data show less evidence for a beat period dependence in the polarization. Given the lower signalto-noise ratio in the total flux measurements, uncertainties on the polarization are larger in this band. Both the linear and circular polarization show near-constant values, at $\approx 1.5$ and $-7 \%$ respectively. While there is some variation with beat phase, this is consistent with the uncertainties on each data point. At this frequency, orbital phases of 0 and 0.5 both miss significant polarized emission features in the orbital light curve, and the beat-folded light curve shows no clear evidence for a beatphase modulation in $1 \mathrm{GHz}$ data near these phases, albeit with large uncertainties on individual data points. We also consider the beat-cycle at orbital phase 0.4-0.6 (where the Stokes $V$ flux is large and negative) in Fig. 16. As expected, the circular polarization is larger than the orbit average, but still shows no clear dependence on beat phase.

\section{Discussion and interpretation}

The strong periodic variability of AR Sco in the optical marked it out as an anomalous source among known white dwarf binaries. Our initial radio observations, obtained with ATCA, demonstrated that these extended to the radio. However they were too short to probe the orbital phase behaviour, and too limited by $u v$-plane coverage and sensitivity to probe short time-scales at high signal to noise.

Here we have demonstrated that the $\mathrm{GHz}$ radio emission of AR Sco is in many ways similar to that observed in the optical. The orbital phase modulation of the total continuum flux is clearly detected across the full range of observed frequencies $(1-10 \mathrm{GHz})$, with the orbital modulation exceeding $50 \%$ of the peak flux. The minimum of the radio lightcurve is also at a similar orbital phase to that observed in the optical, albeit with evidence for radio frequency-dependent variation in the light curve. This provides strong evidence that the radio continuum emission originates from close to the inner face of the M-dwarf, if not from the stellar surface itself. Key orbital phases for the AR Sco system are indicated on a phase diagram for clarity in Fig. 17.

The radio spectral slope $S_{v} \propto v^{0.35}$ is comparable to the selfabsorbed synchrotron slope of $S_{v} \propto v^{0.33}$ derived by Geng et al. (2016) for the $10-1000 \mathrm{GHz}$ region, from data in Marsh et al. (2016), but significantly shallower than the self-absorbed spectral slope, $v^{2}$, they predict for the $1-10 \mathrm{GHz}$ region. Where the total flux peaks, at orbital phase of 0.5 , the spectral index steepens. This may suggest that an additional emission component is contributing to the continuum emission at low radio frequencies $(<5 \mathrm{GHz})$, with the strongest effect where the synchrotron emission spectrum that extends up to the optical is weakest. The orbital light curves shown in Fig. 4 suggest that this lowfrequency emission component peaks twice in the orbital phase, which may imply a bipolar emission source arising from, or near, the (tidally-locked) M-dwarf.

The radio data also confirms strong modulation of the total flux at 5 and $9 \mathrm{GHz}$ on the system beat period and at half this period, with little signal on the spin period. Again the doublepeaked light curve suggests bipolar emission, this time coming from an interaction between the white dwarf and red dwarf, consistent with the extant picture of this source as a nearlyperpendicular rotator, with the white dwarf's polar magnetic fields whipping past the red dwarf twice in each white dwarf 
spin period (Marsh et al. 2016). The very erratic flaring and variation in strength of the signal from pulse to pulse suggests short timescales (a matter of seconds) for variation in the radio emission, suggesting the flaring region is small.

Interestingly these modulations disappear at the lowest frequencies, with the $1.5 \mathrm{GHz}$ data showing no significant evidence for modulation on either the beat or spin frequencies. This hints that the $1.5 \mathrm{GHz}$ flux may arise from a distinct, non-pulsing mechanism in AR Sco, as suggested by the double-peaked orbital lightcurve and the shallow spectral slope.

The exceptional linear polarization seen in the optical is not seen at radio frequencies. The linear polarization of AR Sco in the radio is near constant at $<1 \%$. However, the presence of welldetected circular polarization implies the presence of magnetic fields aligned along the line of sight, and its modulation on both the orbital and beat periods requires further consideration. To first order, the ratio between circular and linear polarization is determined by the Lorentz factor of the emitting electrons, with strong circular polarization implying non-relativistic, cyclotron rather than relativistic synchrotron emission.

This emission source appears to be near-constant, with the beating but unpolarized (in the radio) synchrotron emission superimposed upon it. If so, the polarized emission source likely has a circular polarization well in excess of the measured 5-10\% seen at $9 \mathrm{GHz}$ and is simply being diluted by the stronger, pulsed emission. This is supported by the fact that polarization is also stronger at lower frequencies where the total flux is lower.

Interestingly AR Sco shows very similar orbital phase polarization behaviour to known polars and intermediate polars (IPs) - a class of White Dwarf-M dwarf binaries with which AR Sco shares many of its properties. These sources have typical magnetic fields < $100 \mathrm{MG}$ (Ferrario et al. 2015), slightly lower than that inferred for emission regions in AR Sco from optical polarization (Buckley et al. 2017), and are often characterised by cyclotron emission features in the optical. Several known polars, including VV Puppis (Wickramasinghe \& Meggitt 1982), EXO 03319-2554.2 (Ferrario et al. 1989) and MLS110213 (Silva et al. 2015), show optical circular polarization peaking at about $10 \%$ of the total flux. In IPs, polarization is also observed to modulate on the white dwarf spin period (e.g. Potter et al. 2012) while sources typically show linear polarization comparable to circular, with $L / V \sim 0.2-0.6$ (Wickramasinghe \& Ferrario 2000), comparable to that in $\operatorname{AR~Sco~}(L / V \sim 0.2$ at $5 \mathrm{GHz})$. In the case of both polars and IPs, this is interpreted as a cyclotron emission region arising from single-pole stream-fed accretion onto the surface of the white dwarf.

In AR Sco, despite the striking similarity in the circularly polarized lightcurve, and the circular-to-linear polarization ratio, this model cannot apply in all particulars. There is no sign of accretion, suggesting that the white dwarf cannot contribute in the same manner as in IPs. The much weaker variation on the spin or beat periods, relative to that observed at near-zero orbital phase, also suggests that the circularly-polarized emission arises primarily from the $\mathrm{M}$ dwarf itself, perhaps from steady accretion of magnetically-entrained winds onto polar regions of the red dwarf or from emission in a bow shock region near the surface.

While the white dwarf in AR Sco has a strong magnetic field, the environs of the $M$ dwarf are likely to experience lower field strengths. Their typical surface fields are in the region of 0.1 to $1 \mathrm{kG}$ (although they can be stronger in localised regions) (e.g. Berger 2006). This is comparable to the estimated strength of the white dwarf field at the M dwarf surface $(0.1 \mathrm{kG}$, Katz 2017). These field strengths correspond to non-relativistic cyclotron emission frequencies from 0.3 to $3 \mathrm{GHz}$, which would place the fundamental emission peak for cyclotron flux from the M dwarf within, or just below, our observation frequencies. Our radio observations of AR Sco are thus analogous to optical observations of polars in the sense that the cyclotron frequency of polars is usually in the near-infrared, so that harmonics of order a few are observed in the optical, while we may be seeing the equivalent low order cyclotron/gyro-synchrotron emission from the $\mathrm{M}$ dwarf in AR Sco in the radio.

This emission may be most visible when the irradiated face of the red dwarf (which pulses with strong synchrotron emission on the beat period) is turned out of the line of sight and no longer swamps the lower energy cyclotron emission. As well as the different cyclotron emission site (M dwarf, rather than white dwarf), AR Sco's broad synchrotron spectrum shows that it is distinguished from typical polars by the presence of an extended high energy tail of electrons. These relativistic electrons generate the higher frequency radio, far infrared, infrared, optical and UV emission. If this comes from a region with a $\sim 100 \mathrm{G}$ field, this emission implies electrons with Lorentz factors of order $\sim 1000$, or energies of order a GeV, so it is true synchrotron (rather than cyclotron emission) and we should not expect such high circular polarization. This high energy tail might act to wash out the cyclotron hump structure that one might otherwise expect at radio frequencies if the analogy with optical observations of polars were exact.

\section{Conclusions}

We have obtained high time resolution, interferometric radio spectral imaging and polarization data for AR Sco in the 1.5, 5 and $9 \mathrm{GHz}$ bands, using the Karl G. Jansky Very Large Array. Our main conclusions can be summarized as follows:

- The total radio flux from AR Sco exhibits a power law spectrum between 1 and $10 \mathrm{GHz}$, with a spectral slope of $0.358 \pm$ 0.015 (fit in the range $4-10 \mathrm{GHz}$ ) when flux is integrated over a full orbit.

- The total radio flux is modulated on the $3.56 \mathrm{~h}$ orbital period of the AR Sco binary system. The strength of this modulation is a function of frequency, with the strongest changes seen at $9 \mathrm{GHz}$.

- Emission at 5 and $9 \mathrm{GHz}$ is also modulated on the white dwarf spin-orbit beat period also identified in optical data. Emission at $1.5 \mathrm{GHz}$ shows no apparent beat phase modulation. The fractional pulsation strength decreases with frequency, and is lower in the radio than the optical. The median beat pulse fraction is $\sim 20 \%$ at $9 \mathrm{GHz}$.

- AR Sco shows significant levels of negative circular polarization in a narrow range of orbital phases. The peak of this circularly polarized emission shifts to earlier phases with decreasing frequency, as the fractional strength of the circularly polarized flux increases. Circularly polarized emission also peaks at the minimum of the beat-folded light curve at 5 and $9 \mathrm{GHz}$.

- The linear polarization of AR Sco is far lower in the radio than optical, peaking at a few percent at $1.5 \mathrm{GHz}$, and shows no clear dependence on beat phase at any frequency.

- Emission at $1-10 \mathrm{GHz}$ likely arises from close to or on the surface of the M-dwarf, with no clear evidence for emission from the white dwarf.

- The 1-10 GHz frequency regime appears to mark a transition between different dominant emission regions and mechanisms, likely breaking from the synchrotron power-law emission that extends up to the optical towards a non-relativistic, 
circularly polarized cyclotron emission mechanism at low frequencies.

In conclusion, the radio observations of AR Sco have revealed a hitherto unseen, non-relativistic electron emission component at low radio frequencies. The system nonetheless remains a challenging source to interpret, and would benefit from further observations and theoretical study. Simultaneous observations at multiple frequencies in the radio may be necessary to fully disentangle flaring and orbit-to-orbit variation from frequency dependent shifts in behaviour. This presents significant technica challenges, both in terms of the shortage of arrays with multiband receivers, and the difficulty of obtaining sufficient signal to noise and support in the $u v$-plane for the short integrations required.

Acknowledgements. T.R.M., P.J.W. and D.S. are supported by UK Science and Technology Facilities Council (STFC) Consolidated Grant ST/P000495/1 The research leading to these results has received funding from the European Research Council under the European Union's Seventh Framework Programme (FP/2007-2013)/ERC Grant Agreement no. 320964 (WDTracer). The National Radio Astronomy Observatory is a facility of the National Science Foundation operated under cooperative agreement by Associated Universities, Inc.

\section{References}

Berger, E. 2006, ApJ, 648, 629

Buckley, D. A. H., Meintjes, P. J., Potter, S. B., Marsh, T. R., \& Gänsicke, B. T. 2017, Nat. Astron., 1, 0029

Ferrario, L., Wickramasinghe, D. T., Bailey, J., Tuohy, I. R., \& Hough, J. H. 1989, ApJ, 337, 832

Ferrario, L., de Martino, D., \& Gänsicke, B. T. 2015, Space Sci. Rev., 191, 111

Geng, J.-J., Zhang, B., \& Huang, Y.-F. 2016, ApJ, 831, L10

Katz, J. I. 2017, ApJ, 835, 150

Littlefield, C., Garnavich, P., Kennedy, M., et al. 2017, ApJ, 845, L7

Lomb, N. R. 1976, Ap\&SS, 39, 447

Marcote, B., Marsh, T. R., Stanway, E. R., Paragi, Z., \& Blanchard, J. M. 2017, A\&A, 601, L7

Marsh, T. R., Gänsicke, B. T., Hümmerich, S., et al. 2016, Nature, 537, 374

Potter, S. B., Romero-Colmenero, E., Kotze, M., et al. 2012, MNRAS, 420 , 2596

Press, W. H., \& Rybicki, G. B. 1989, ApJ, 338, 277

Scargle, J. D. 1982, ApJ, 263, 835

Silva, K. M. G., Rodrigues, C. V., Oliveira, A. S., et al. 2015, MNRAS, 451, 4183

Stockman, H. S., Schmidt, G. D., \& Lamb, D. Q. 1988, ApJ, 332, 282

Warner, B. 2002, in Classical Nova Explosions, eds. M. Hernanz, \& J. José, AIP Conf. Proc. 637, 3

Wickramasinghe, D. T., \& Ferrario, L. 2000, PASP, 112, 873

Wickramasinghe, D. T., \& Meggitt, S. M. A. 1982, MNRAS, 198, 975 